\title{
CORRIGENDUM
}

\section{Ultrastable glasses from in silico vapour deposition}

Sadanand Singh, M. D. Ediger and Juan J. de Pablo

Nature Materials 12, 139-144 (2013); published online 6 January 2013; corrected after print 13 May 2014.

We reported the formation of stable binary glass films by vapour deposition. The results were generated on relatively small samples. Simulations of larger systems carried out after the publication of this work have revealed that a significant fraction of the stabilization effect reported can be attributed to composition inhomogeneities that arise near the interfaces of the films. An analysis of these effects is reported in J. Chem. Phys. 139, 144505 (2013). In particular, the extrapolated cooling rates that would be required to generate ordinary glasses with inherent-structure energies comparable to those of vapour-deposited glasses are approximately 2-3 orders of magnitude smaller than the cooling rates used in traditional liquid-cooling simulations. This number is in contrast to the 19 ordersof-magnitude difference inferred from the smaller samples. Configuration files for the larger systems and the corresponding characterizations are available, and can be provided on request. Furthermore, in the Supplementary Information, in Table T1, the values of the energies $E_{\mathrm{IS}}$ and $\langle U\rangle$, and the $Q_{6}$ parameter corresponding to ordinary glasses, and in Table T2, the $\langle U\rangle$ values, have been corrected to reflect the reported densities. Ivan Lyubimov, from the Institute of Molecular Engineering, University of Chicago, performed the calculations that led to this correction. 